\title{
Severe emphysematous cystitis: Outcome after seven days of antibiotics
}

\author{
Maria Sereno*, César Gómez-Raposo, Gerardo Gutiérrez- \\ Gutiérrez, Miriam López-Gómez, Enrique Casado
}

\begin{abstract}
We present the case of a 70-year-old woman with emphysematous cystitis. She was a diabetic patient and she was on chemotherapy treatment for a breast cancer. She complaint of severe asthenia and pain in her right lower extremity, but no fever or urinary symptoms. A computed tomography (CT) scan was suggestive of severe emphysematous cystitis. Emphysematous cystitis is a rare clinically entity, more commonly seen in diabetic, immunocompromised patients. A conservative treatment approach using antibiotics and bladder catheterization is typically successful, with a complication rate less than $20 \%$.
\end{abstract}

KEYWORDS: emphysematous cystitis

\section{CASE REPORT}

A 70-year-old woman with diabetes diagnosed of breast cancer T1N1 several months ago treated with surgery and chemotherapy was admitted to our hospital because of severe asthenia and pain in her right lower extremity. She did not refer either urinary symptoms or fever. She was still on chemotherapy (last cycle 20 days ago). Glucose levels were not well controlled (mean over $300 \mathrm{mg} /$ dl) due to corticoids use as well as chemotherapy pre-medication. More than $1 \times 106$ colonies of E.coli grew from her urine and blood cultures. A computed tomography (CT) scan demonstrated a thickened, trabeculated bladder wall containing pockets of gas (Figure A). These findings were suggestive of severe emphysematous cystitis (1). Intravenous Piperacillin-tazobactam was administrated with bladder catheterization (2). After one week of antibiotics, the patient improved clinically and another CT was taken, showing a considerable reduction of the gas inside bladder wall (Figure B).

\footnotetext{
*To whom correspondence should be addressed: María Sereno Moyano

Julio Rey pastor Street, n 6, block 6, 4B, San Sebastian de los Reyes Madrid, Spain 28702

Telephone +34649002612

Email: Mariasereno75@gmail.com
}

The outcome was favourable after three weeks on antibiotic treatment. So far, there is no evidence of relapse and she is on follow-up in Oncology Department

\section{DISCUSSION}

Despite belonging to a low percentage of all the urinary tract infections, gas-producing infections are relevant as they may lead to death. Three categories of this type of infections are recognized: emphymatous pyelonephritis, emphymatous pyelitis or emphymatous cystitis.

Emphymatous pyelonephritis is a necrotic infection. The gas is produced in the renal and perirenal parenchyma and $90 \%$ of the cases are reported in diabetic patients. The delay on the appropriate therapy contributes to a high mortality rate, in some case series up to $80 \%$ (3).

Emphymatous cystitis is a rare disease that is mainly diagnosed in diabetic and immunocompromised patients. It is also reported to be in association with neurogenic bladder, obstruction of the urinary tracts, catheter use and chronic infections of the urinary tract. 

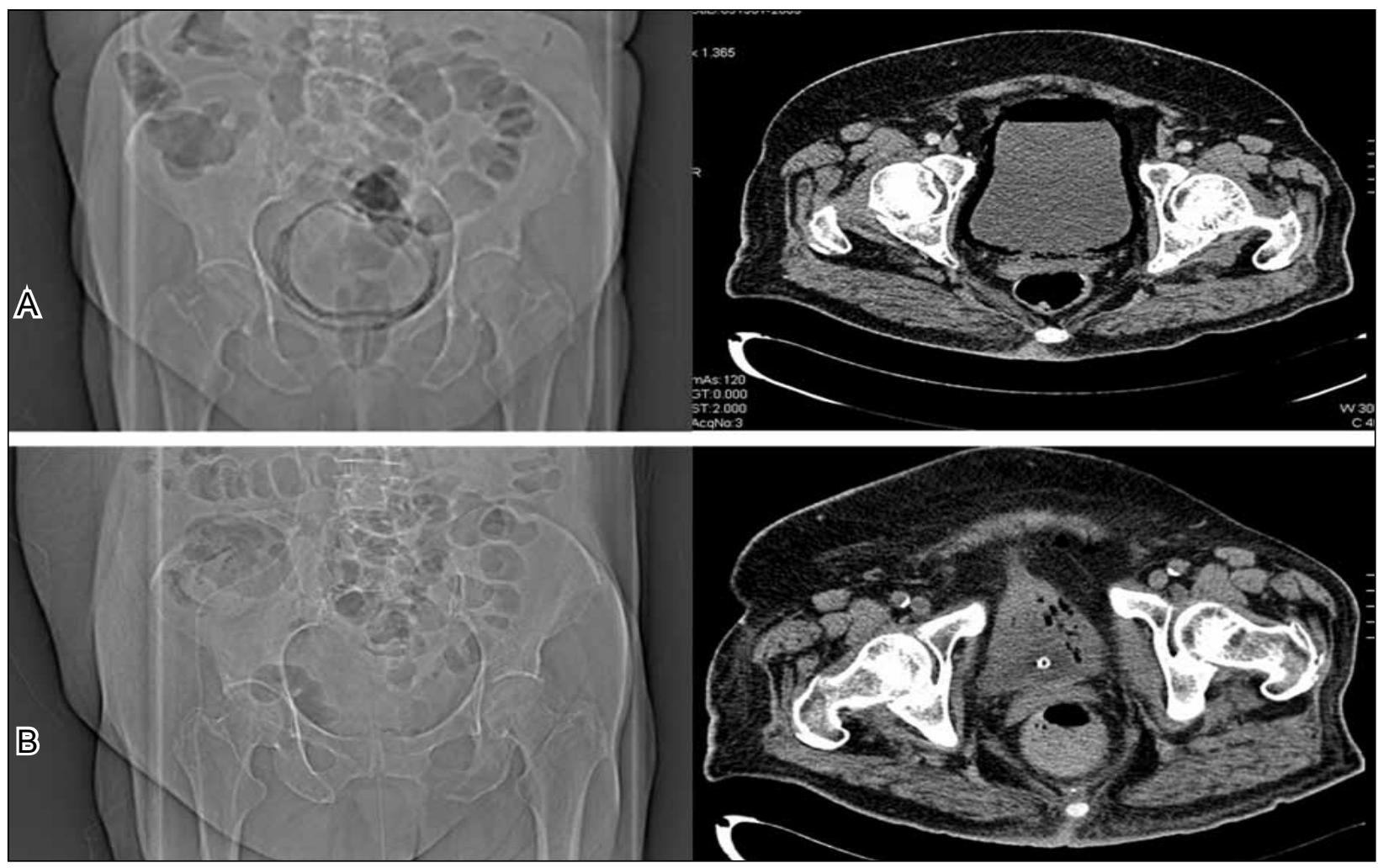

The microorganisms most often involved in this infection are Escherichia coli and Klebsiella pneumonia and the less common are Enterobacter, Proteus, Streptococci and Candida (4). Though, bacteria are the most common agent, fungi may also be responsible for this clinical picture (5).

The exact mechanism by which the gas is produced in the emphysematous infection is not quite clear. In diabetic patients, one of the reasons seems to be the production of $\mathrm{CO} 2$ by the microorganisms through the fermentation of glucose, which occurs when the glucose concentration is high. Since the emphymatous infections may occur in nondiabetic patients, it has been suggested that the urinary lactulose and tissue proteins may be useful as substrate to the gas production (6).

Another factor that may help in this process is the impaired transportation of gas due to the local inflammation or some kind of obstructive process increasing the local pressure and decreasing the circulation. This may involve tissue necrosis which becomes a good culture for pathogens to produce gas (7).

The most common clinical features are fever and abdominal pain along with dysuria, haematuria and pneumaturia (8). In our patient, the presentation was unusual with pain in her right lower extremity, but without urinary symptoms.
The diagnosis is provided by radiographic image (X-ray or CT scan). The most obvious radiographic clues are small pockets of gas in the mucous membrane of the bladder, as we can see in this picture.

Other causes for the presence of air in the bladder such as fistula with the intestine or vagina, after trauma, cancer or instrumentation, have to be excluded (3).

The appropriate treatment involves endovenous antibiotic therapy with broad-spectrum such as fluoroquinolones, penicillin with inhibitor of the beta-lactamases (imipenem, ticarcillin/clavulanate) or third-generation cefalosporins. Antifungal agents may be used (systemic or intravesical), if a fungus infection is reported (6). The full recovery from any infection with gas production depends on early diagnosis plus correction of the subjacent causes, glycemic control, long-term therapeutic with antibiotic therapy (3 to 6 weeks) and surgery, if required (3). Our patient had a fast response to intravenous antibiotic. After 7 days on treatment the radiological and clinical features had improved considerably. The reported case shows the seriousness and the atypical presentation that this infection may assume. The diagnosis was made indeed on a diabetic patient in an unusual clinical situation. The diabetes mellitus and the poor glycemic control are 
the main risk factors for this type of infection. The agent isolated from this patient, the Escherichia coli is reported as the most common. The empiric broad-spectrum antibiotic (piperacillin/tazobactam) used for the nosocomial urinary tract infection was found to be highly effective.

\section{REFERENCES}

1. Thomas AA, Lane BR, Thomas AZ; et al. Emphysematous cystitis: a review of 135 cases. BJU Int. 2007;100: 17-20

2. Bittleman DB. Cystitis emphysematosa. N Y State J Med 1991; 91:110-1.

3. Kumarhttp A, Turney JH, Brownjohn MA; et al. Unusual bacterial infections of the urinary tract in diabetic patients-rare but frequently lethal. Nephrol Dial Transplant 2001;16:1062-5
4. Nemati E, Basra R, Fernandes $\mathrm{H}$; et al. Emphysematous cystitis. Nephrology Dialysis Transplantation 2005;20:6523.

5. Decambre M, Albertsen P, Rutchik S. Emphysematous Cystitis: Caveats of Complex Presentations. Infect in Uro 2002; 15:19-21.

6. Galiano Baena JF, Caballero Romeu JP, Galán Llopis JA, Leivar Tamayo A, Lobato Encinas JJ. Emphysematous cystitis. Case report and review of literature]. Actas Urol Esp. 2008; 32:948-50.

7. Grupper M, Kravtsov A, Potasman I. Emphysematous Cystitis: Illustrative Case Report and Review of the Literature. Medicine 2007; 86: 47-53.

8. Reese AC, Stoller ML. Emphysematous Cystitis. Urology. 2009; 23 [Epub ahead of print]

María Sereno (PhD, MD) is a medical oncologist in Infanta Sofía Hospital. She has a MBA in Molecular Oncology and she is conducting research in lung cancer.

César Gómez- Raposo (MD) is a medical oncologist in Infanta Sofía Hospital. He is conducting research in breast cancer and discovering predictors of response to new drugs.

Gerardo Gutiérrez-Gutiérrez (MD) is a neurologist in Infanta Sofía Hospital. He is conducting research in neuromuscular disease and neuroprotection, mainly in neuropathy induced by chemotherapy.

Miriam López-Gómez (MD) is a medical oncologist in Infanta Sofía Hospital. She is conducting research in colon cancer and the behavior of cancer cells in liver metastasis.

Enrique Casado Sáenz (PhD, MD) he is the chief of Oncology Department, Infanta Sofía Hospital. He is focussed in rectal cancer research and he is mainly interested in angiogenic phenomenon and predictors of response to antiangiogenic drugs. 